\title{
A CLASS OF FACTORABLE TOPOLOGICAL ALGEBRAS
}

\author{
by E. ANSARI-PIRI
}

(Received 29th February 1988, revised 18th July 1988)

\begin{abstract}
The famous Cohen factorization theorem, which says that every Banach algebra with bounded approximate identity factors, has already been generalized to locally convex algebras with what may be termed "uniformly bounded approximate identities". Here we introduce a new notion, that of fundamentality generalizing both local boundedness and local convexity, and we show that a fundamental Fréchet algebra with uniformly bounded approximate identity factors. Fundamentality is a topological vector space property rather than an algebra property. We exhibit some non-fundamental topological vector space and give a necessary condition for Orlicz space to be fundamental.
\end{abstract}

1980 Mathematics subject classifications (1985 Revision): 46H

\section{Introduction}

It has been remarked by Zelazko [9] that the famous Cohen factorization theorem extends in a straightforward way to locally bounded algebras with bounded approximate identities. Furthermore, in [3], it has also been generalized to locally convex, complete metrizable topological algebras (locally convex $F$-algebras) with uniformly bounded approximate identities. In [3] it is asked whether it is possible to prove the factorization theorem in the absence of local convexity. In this note we give an affirmative answer to this question. We introduce a new notion, that of "fundamentality" generalizing both local boundedness and local convexity, and we will show that a fundamental $F$-algebra with uniformly bounded approximate identity can be factorized; therefore this notion unifies the earlier results.

In fact, fundamentality is a topological vector space property rather than an algebra property. In Section 2, we give some definitions and some related results. In Section 3, we exhibit some non-fundamental topological vector spaces and give a necessary condition for a certain class of spaces, namely, Orlicz spaces, to be fundamental, and finally in Section 4 we prove that an $F$-algebra with uniformly bounded approximate identity which is fundamental can be factorized. For background information on approximate identities and factorization we refer the reader to [4]. Earlier work on these ideas in LMC algebras may be found in [2] and [8].

I should like to thank my supervisor, Dr P. G. Dixon, for his advice and encouragement during the preparation of this paper. My thanks are also due to the Ministry of Culture and Higher Education of the Islamic Republic of Iran and Guilan University for financial support. 


\section{Definitions and related results}

Throughout the paper we write $\mathbb{F}$ for the scalar field, which may be either the real or the complex numbers, and in this section $A$ will denote a topological vector space over $\mathbb{F}$.

2.1. $A$ is said to be a fundamental topological vector space if there exists $b>1$ such that for every sequence $\left(a_{n}\right)$ of $A$ the convergence of $b^{n}\left(a_{n+1}-a_{n}\right)$ to zero in $A$ implies that $\left(a_{n}\right)$ is a Cauchy sequence.

2.2. Let $E$ be any set. $A$ is said to be uniformly fundamental on $E$ if there exists $b>1$ such that for every sequence $\left(f_{n}\right)$ of functions from $E$ into $A, b^{n}\left(f_{n+1}(t)-f_{n}(t)\right) \rightarrow 0$ uniformly on $E$ implies that $\left(f_{n}\right)$ is uniformly Cauchy on $E$. $A$ is said to be a uniformly fundamental topological vector space if it is uniformly fundamental on every set $E$.

2.3. A topological algebra $A$, whose underlying topological vector space is fundamental (uniformly fundamental), is called a fundamental (uniformly fundamental) topological algebra.

It is easy to check that every locally convex and every locally bounded space is a uniformly fundamental topological vector space (for a locally bounded space, it suffices to see that its topology can be defined by an $\alpha$-norm [6, p. 40]). On the other hand, if $B$ is a locally convex but not locally bounded space, and $C$ is a locally bounded but not locally convex space, then $B \oplus C$ is a uniformly fundamental topological vector space which is not locally convex and not locally bounded.

Proposition 2.4. Let $A$ be a fundamental topological vector space, then for every $c>1$ and every sequence $\left(a_{n}\right)$ of $A$, the convergence of $c^{n}\left(a_{n+1}-a_{n}\right)$ to zero in $A$ implies that $\left(a_{n}\right)$ is a Cauchy sequence. For the uniformly fundamental case the corresponding result holds.

Proof. Let Definition 2.1 be satisfied with the constant $b>1$, and for some $k \in \mathbf{Z}^{+}$, $c^{2^{k}}>b$. Then obviously Definition 2.1 is satisfied with $c^{2^{k}}$. Suppose $\left(a_{n}\right)$ is any sequence of $A$ with $\left(c^{2^{k-1}}\right)^{n}\left(a_{n+1}-a_{n}\right) \rightarrow 0$, then $\left(c^{2^{k-1}}\right)^{2 n}\left(a_{2 n+1}-a_{2 n}\right) \rightarrow 0$ and $\left(c^{2^{k-1}}\right)^{2 n+1}\left(a_{2 n+2}-a_{2 n+1}\right) \rightarrow 0$ which implies that $\left(c^{2^{k}}\right)^{n}\left(a_{2 n+2}-a_{2 n}\right) \rightarrow 0$ and so $\left(a_{2 n}\right)$ is Cauchy. Since also $a_{n+1}-a_{n} \rightarrow 0$, we get that $\left(a_{n}\right)$ is Cauchy. Therefore, the fact that Definition 2.1 is satisfied with $c^{2^{k}}$ implies that it is also satisfied with $c^{2^{k-1}}$. Now by repeating, if necessary, the assertion is proved.

Lemma 2.5. If $A$ is uniformly fundamental on $\mathrm{Z}^{+}$, then $A$ is uniformly fundamental on every set $E$.

Proof. If $\left(f_{n}\right)$ is uniformly Cauchy on countable subsets of $E$, then $\left(f_{n}\right)$ is uniformly Cauchy on $E$. 
Proposition 2.6. If $A$ is complete, metrizable, and fundamental, then it is uniformly fundamental on every set $E$.

Proof. Let Definition 2.1 be satisfied with a constant $b>1$. Let $X_{1}$ (respectively $X_{2}$ ) be the space of all sequences (respectively, bounded double sequences) of $A$ converging to zero in $A$ with a topology defined by $\left\{\hat{U}_{n}\right\}_{n=1}^{\infty}$ (respectively $\left\{\hat{U}_{n}\right\}_{n=1}^{\infty}$ ) as a neighbourhood base at zero where $\mathscr{B}=\left\{U_{n}\right\}_{n=1}^{\infty}$ is a neighbourhood base at zero in $A$ and for each $n \in \mathbf{Z}^{+}, \hat{U}_{n}$ and $\hat{U}_{n}$ are defined by

$$
\begin{gathered}
\hat{U}_{n}=\left\{\left(x_{j}\right)_{j=1}^{\infty} \in X_{1}: x_{j} \in U_{n} \forall j \in \mathbf{Z}^{+}\right\} \\
\hat{U}_{n}=\left\{\left(x_{p, q}\right)_{p, q=1}^{\infty} \in X_{2}: x_{p, q} \in U_{n} \forall p, q \in \mathbf{Z}^{+}\right\}
\end{gathered}
$$

For each sequence $\left(x_{n}\right) \in X_{1}$, put $f_{n}=\sum_{j=1}^{n} b^{-j} x_{j}$. The fundamentality of $A$ implies that the double sequence $\left(f_{p}-f_{q}\right)_{p, q=1}^{\infty} \in X_{2}$, Define $T: X_{1} \rightarrow X_{2}$ by $T\left(\left(x_{n}\right)\right)=\left(f_{p}-f_{q}\right)_{p, q=1}^{\infty}$. By the closed graph theorem, $T$ is continuous.

Let $\left(f_{n}\right)$ be a sequence of functions from $\mathrm{Z}^{+}$into $A$ such that $b^{n}\left(f_{n+1}(k)-f_{n}(k)\right) \rightarrow 0$ uniformly on $\mathbf{Z}^{+}$and let $W \in \mathscr{B}$. Take $V \in \mathscr{B}$ with $T(\hat{V}) \subseteq \hat{W}$. There exists $N_{0} \in \mathbf{Z}^{+}$ such that $n>N_{0}$ implies that for each $k \in \mathbf{Z}^{+}, \quad b^{n}\left(f_{n+1}(k)-f_{n}(k)\right) \in V$. Put $x_{n}^{k}=b^{N_{0}+n}\left(f_{N_{0}+n+1}(k)-f_{N_{0}+n}(k)\right)$ then for each $k \in \mathbf{Z}^{+},\left(x_{n}^{k}\right)_{n=1}^{\infty} \in \hat{V}$ and therefore for each $\dot{k} \in \mathbf{Z}^{+}, T\left(\left(x_{n}^{k}\right)_{n=1}^{\infty}\right) \in \hat{\hat{W}}$. Therefore the sequence $\left(f_{n}\right)$ is uniformly Cauchy on $\mathbf{Z}^{+}$. Now by Lemma 2.5 the proposition is proved.

Proposition 2.7. If $\Lambda$ is a directed set and $\left(A_{\lambda}\right)_{\lambda \in \Lambda}$ is a projective family of uniformly fundamental topological algebras, then so is $\lim A_{\lambda}$.

The proof is straightforward.

Proposition 2.8. Let $A$ be a metrizable uniformly fundamental topological vector space and $M \subseteq A$ a closed vector subspace then the quotient space $A / M$ is uniformly fundamental.

The proof is easy and omitted.

\section{Non-fundamental topological vector spaces}

There are many topological vector spaces which are not fundamental. For example, the space $\mathscr{M}([0, \infty))$ of all measurable functions on $[0, \infty)$, with the convergence in measure topology is not fundamental.

Let $g:[0, \infty) \rightarrow \mathbb{R}$ be an Orlicz function $[5$, p. 29); we define the Orlicz space

$$
L^{g}([0, \infty))=\left\{f \in \mathscr{M}([0, \infty)): \int_{0}^{\infty} g(|f(t)|) d t<\infty\right\}
$$


with topology given by the neighborhood base $\left\{U_{n}\right\}_{n=1}^{\infty}$ where

$$
U_{n}=\left\{f \in L^{\ell}([0, \infty)): \int_{0}^{\infty} g(|f(t)|) d t<1 / n\right\}
$$

Then we have:

Proposition 3.1. A necessary condition for the Orlicz space $L^{g}([0, \infty))$ to be fundamental is that for each $b>1$ and each $0 \leqq \alpha<1$, the sequence $\left(g\left(b^{2 n}\right) / n^{\alpha} g\left(b^{n}\right)\right)_{n=1}^{\infty}$ be unbounded.

Proof. Suppose that for some $0 \leqq \alpha<1$ and some $b>1,\left(g\left(b^{2 n}\right) / n^{\alpha} g\left(b^{n}\right)\right)_{n=1}^{\infty}$ is a bounded sequence. Take $\gamma \in(\alpha, 1)$ and define $\mu_{0}=0$, and $\mu_{n}=1 / n^{\gamma} g\left(b^{n-1}\right)$ for $n \geqq 1$. Now, if we define $f_{n}:[0, \infty) \rightarrow[0, \infty)$ by

$$
f_{n}(t)= \begin{cases}b^{k} & \text { if } \sum_{i=0}^{k} \mu_{i} \leqq t<\sum_{i=0}^{k+1} \mu_{i} \text { for } k=0, \ldots, n-1 \\ 0 & \text { otherwise }\end{cases}
$$

then

$$
\int_{0}^{\infty} g\left(\left|b^{n}\left(f_{n+1}(t)-f_{n}(t)\right)\right|\right) d t=g\left(b^{2 n}\right) /(n+1)^{\gamma} g\left(b^{n}\right) \rightarrow 0
$$

but

$$
\int_{0}^{\infty} g\left(\left|f_{m}(t)-f_{n}(t)\right|\right) d t=\sum_{k=n}^{m-1} g\left(b^{k}\right) \mu_{k+1} \nrightarrow 0 .
$$

Example 3.2. Let $g(t)=\log ^{\beta}(1+t)$ for $t \in[0, \infty)$ and $\beta>0$. Then, obviously for each $b>1, \quad\left(g\left(b^{2 n}\right) / g\left(b^{n}\right)\right)_{n=1}^{\infty}$ is a bounded sequence and therefore $L^{g}([0, \infty))$ is not fundamental.

\section{Factorization in fundamental $F$-algebras}

In this section, we prove that if an $F$-algebra has a uniformly bounded approximate identity, even in the absence of local convexity and local boundedness it may factorize.

Let $A$ be a complete, metrizable topological algebra and $\left\{U_{n}\right\}_{n=1}^{\infty}$ be a countable neighbourhood base at zero such that each $U_{n}$ is closed, balanced, $U_{n+1} \subseteq U_{n}$ and $U_{n+1}^{2} \subseteq U_{n}\left(n \in Z^{+}\right)$. Let $\tilde{A}$ be the unitization of $A\left(\left[1\right.\right.$, p. 15]). If we define $\tilde{U}_{n}=$ $U_{n}+B[0,1 / n]$, where $B[0,1 / n]=\{\alpha \in \mathbb{F}:|\alpha| \leqq 1 / n\}$, then $\left\{\tilde{U}_{n}\right\}_{n=1}^{\infty}$ is a countable closed, balanced neighbourhood base at zero for $\tilde{A}$ such that $\tilde{A}$ itself becomes a metrizable complete topological algebra with identity. We have $\tilde{U}_{n+1} \cdot U_{n+1} \subseteq U_{n}+U_{n}\left(n \in \mathbf{Z}^{+}\right)$. As 
in [3], by a uniformly bounded left approximate identity in $A$, we shall mean a net $\left(e_{\lambda}\right)_{\lambda \in \Lambda}$ such that $e_{\lambda} a \rightarrow a$ for every $a \in A$ and there exists $k>0$ such that $\left\{\left(k^{-1} e_{\lambda}\right)^{n}: \lambda \in \Lambda, n \in Z^{+}\right\}$ is bounded in $A$; then we say $k$ is a uniform bound for $\left(e_{\lambda}\right)_{\lambda \in \Lambda}$. Also, by a left Fréchet $A$-module, we shall mean a left $A$-module $X$ which is a complete, metrizable topological vector space, such that the multiplication map $(a, x) \rightarrow a x: A \times X \rightarrow X$ is continuous. We shall say that $X$ is essential if $A$ has a uniformly bounded left approximate identity $\left(e_{\lambda}\right)_{\lambda \in \Lambda}$ such that $e_{\lambda} x \rightarrow x$ for all $x \in X$. We shall let $d$ denote a translation-invariant metric defining the same topology on $X$, and we write $|x|=d(x, 0)(x \in X)$.

Theorem 4.1. Let $A$ be fundamental and $X$ be an essential left Fréchet A-module. Then for each $x \in X$ an each $\varepsilon>0$, there exists $a \in A$ and $y \in X$ such that $x=a y, y \in \overline{A x}$, and $d(x, y)<\varepsilon$.

Proof. Let $\left(e_{\lambda}\right)_{\lambda \in \Lambda}$ be the uniformly bounded left approximate identity in $A$ with a uniform bound $k>1$, and take $0<\rho / 1+\rho<1 / 2 k$, and $1<b<1+\rho$. For $j \in Z^{+}$, let $M_{j}>j$ be such that for each $n \in \mathbf{Z}^{+}$and $\lambda \in \Lambda, k^{-n} e_{\lambda} \in M_{j} U_{j}$. Then we have $\left(\rho(1+\rho)^{-1} e_{\lambda}\right)^{n} \in 2^{-n} M_{j} U_{j}$ and therefore $\left(\rho(1+\rho)^{-1} e_{\lambda}\right)^{n} \rightarrow 0$ uniformly on $\Lambda$. Now, if we put $S_{n}(\lambda)=\sum_{i=1}^{n}\left(\rho(1+\rho)^{-1} e_{\lambda}\right)^{i}$, since $b<1+\rho<2$, then, for sufficiently large $n$,

$$
S_{n+1}(\lambda)-S_{n}(\lambda)=\left(\rho(1+\rho)^{-1} e_{\lambda}\right)^{n+1} \in 2^{-n-1} M_{j} U_{j} \subseteq b^{-n} U_{j}
$$

and so $b^{n}\left(S_{n+1}(\lambda)-S_{n}(\lambda)\right) \rightarrow 0$ uniformly on $\Lambda$. By Proposition 2.6 , it follows that the sequence $\left(S_{n}\right)$ in $\tilde{A}$ is uniformly Cauchy on $\Lambda$, and then, the series $\sum_{n=0}^{\infty}\left(\rho(1+\rho)^{-1} e_{\lambda}\right)^{n}$ converges uniformly on $\Lambda$. Therefore $\left(1+\rho-\rho e_{\lambda}\right)$ is invertible in $\tilde{A}$ and for each $\lambda \in \Lambda$ we have

$$
\left(1+\rho-\rho e_{\lambda}\right)^{-1}=(1+\rho)^{-1} \sum_{n=0}^{\infty}\left(\rho(1+\rho)^{-1} e_{\lambda}\right)^{n} \in \tilde{A}
$$

Now for $j \in \mathbf{Z}^{+}$, take $j_{1}\left(=j_{1}(j)\right)$ such that $\tilde{U}_{j_{1}}+\tilde{U}_{j_{1}} \subseteq \tilde{U}_{j}$. Let $k_{j} \in \mathbf{Z}^{+}$be such that for each $\lambda \in \Lambda, \sum_{n=k_{j}+1}^{\infty}\left(\rho(1+\rho)^{-1} e_{\lambda}\right)^{n} \in \tilde{U}_{j_{1}}$. Take $j_{0} \in \mathbf{Z}^{+}$(depending only on $j$ ) such that $\tilde{U}_{j_{1}} \supseteq \tilde{U}_{j_{0}}+\cdots+\tilde{U}_{j_{0}}\left(\left(k_{j}+1\right)\right.$ summands $)$.

We have

$$
1+\left(\rho(1+\rho)^{-1} e_{\lambda}\right)^{1}+\cdots+\left(\rho(1+\rho)^{-1} e_{\lambda}\right)^{k_{j}} \in M_{j_{0}} \tilde{U}_{j 0}+\cdots+M_{j_{0}} \tilde{U}_{j 0},
$$

and then $\sum_{n=0}^{\infty}\left(\rho(1+\rho)^{-1} e_{\lambda}\right)^{n} \in M_{j 0} \tilde{U}_{j}$. Thus for each $j \in \mathbf{Z}^{+}$, there exists $j_{0}(j)$ such that

$$
\left(1+\rho-\rho e_{\lambda}\right)^{-1} \in(1+\rho)^{-1} M_{j 0} \tilde{U}_{j} \quad(\forall \lambda \in \Lambda) .
$$

Now take $e_{1} \in A$ such that $\left|\rho x-\rho e_{1} x\right|<\varepsilon / 2$. Suppose $e_{1}, e_{2}, \ldots, e_{n}$ have been chosen. Define $t_{n}=\left(1+\rho-\rho e_{1}\right) \ldots\left(1+\rho-\rho e_{n}\right), h_{n}=t_{n}^{-1} \in \tilde{A}, a_{n}=t_{n}^{-1}-(1+\rho)^{-n} \in A$ and $y_{n}=$ $t_{n} x \in X$. Now choose $e_{n+1} \in A$ such that

$$
e_{n+1} a_{n}-a_{n} \in b^{-n} U_{n}
$$


and

$$
\left|\rho t_{n} x-\rho t_{n} e_{n+1} x\right|<\varepsilon 2^{-n-1}
$$

By definition we have

$$
a_{n+1}-a_{n}=(1+\rho)^{-n}\left(1+\rho-\rho e_{n+1}\right)^{-1} \rho(1+\rho)^{-1} e_{n+1}+\left(1+\rho-\rho e_{n+1}\right)^{-1} \rho\left(e_{n+1} a_{n}-a_{n}\right)
$$

and so, for all $j \in \mathbf{Z}^{+}$,

$$
a_{n+1}-a_{n} \in(1+\rho)^{-n-1} M_{(j+1) 0} \tilde{U}_{j+1} \cdot M_{j+1} U_{j+1}+(1+\rho)^{-1} M_{(j+1)_{0}} \tilde{U}_{j+1} \cdot \rho b^{-n} U_{n}
$$

and then for sufficiently large $n$ we have

$$
(1+\rho)^{-n-1} M_{(j+1) 0} M_{j+1} \tilde{U}_{j+1} U_{j+1} \subseteq b^{-n}\left(U_{j}+U_{j}\right)
$$

and

$$
\rho(1+\rho)^{-1} M_{(j+1) 0} \tilde{U}_{(j+1)} b^{-n} U_{n} \subseteq b^{-n}\left(U_{j}+U_{j}\right)
$$

which implies that $\left(a_{n}\right)$ is a Cauchy sequence in $A$. Let $a=\lim _{n} a_{n} \in A$. Since by $(*),\left(y_{n}\right)$ is also a Cauchy sequence and $x=h_{n} y_{n}$ we have clearly $x=a y$ with $y=\lim _{n} y_{n} \in \overline{A x} \subseteq X$ and $d(x, y)<\varepsilon$.

Corollary 4.2. Let $A$ be as in Theorem 4.1, then for every sequence $\left(x_{n}\right)$ of $A$ converging to zero, there exists an element $a \in A$ and a sequence $\left(y_{n}\right)$ of $A$ converging to zero such that $x_{n}=a y_{n}$ for all $n \in \mathbf{Z}^{+}$.

Proof. Let $X_{1}$ be as in Proposition 2.6 and the neighbourhood base $\left\{U_{n}\right\}_{n=1}^{\infty}$ of $A$ be chosen as above, then $\left\{\hat{U}_{j}\right\}_{j=1}^{\infty}$ is a neighbourhood base at zero in $X_{1}$ making $X_{1}$ a Fréchet left $A$-module where $\hat{U}_{j}$ is defined by

$$
\hat{U}_{j}=\left\{\left(z_{n}\right) \in X_{1}: z_{n} \in U_{j} \forall n \in \mathbf{Z}^{+}\right\}
$$

Since $X_{1}$ is also essential, the corollary follows by applying Theorem 4.1 with $X=X_{1}$.

Corollary 4.3. Let $A$ be as in Theorem 4.1 with a continuous involution *: $A \rightarrow A$, then, every positive functional on $A$ is continuous.

Proof. The proof is just as in Theorem 4.3 in [3]. 


\section{REFERENCES}

1. F. F. Bonsall and J. Duncan, Complete Normed Algebras (Springer-Verlag, Berlin, 1973).

2. I. G. CRAw, Factorization in Fréchet-algebras, J. London Math. Soc. 44 (1969), 607-611.

3. P. G. Dixon, Automatic continuity of positive functionals on topological involution algebras, Bull. Austral. Math. Soc. 23 (1981), 265-281.

4. R. S. Doran and J. Wichmann, Approximate Identities and Factorization in Banach Modules (Lecture Notes in Mathematics 768, Springer-Verlag, Berlin, 1979).

5. N. J. Kalton, N. T. PecK and James W. RoberTs, An F-space Sampler (London Mathematical Society Lecture Note series, 89, Cambridge University Press, Cambridge, 1984).

6. A. Mallios, Topological Algebras, Selected Topics (Mathematical Studies, 124, NorthHolland, Amsterdam, 1986).

7. A. P. Robertson and Wendy Robertson, Topological Vector Spaces (Cambridge University Press, Cambridge, Second Edition 1973).

8. M. K. Summers, Factorization in Fréchet modules, J. London Math. Soc. (2), 5 (1972), 243-248.

9. W. Żelazko, Banach Algebras (PWN-Polish Scientific Publishers, Warszawa 1973).

Department of Pure Mathematics

Faculty OF ScIENCE

GUILAN UNIVERSITY

P.O. Box 451

Rasht, Iran 